\title{
Evaluation of a random displacement model for predicting particle escape from
} canopies using a simple eddy diffusivity model Elizabeth Follett $^{a \star}$, Marcelo Chamecki ${ }^{b}$, Heidi Nepf ${ }^{a}$

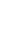

(1)
a. Department of Civil and Environmental Engineering, Massachusetts Institute of Technology, 77 Massachusetts Avenue Room 1-290, Cambridge, MA, 02139, USA E-mail: emf@mit.edu,hmnepf@mit.edu

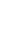

b. Department of Meteorology, The Pennsylvania State University, 503 Walker Building,

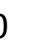
University Park, PA, 16802, USA E-mail: chamecki@psu.edu

12

3 *Corresponding author: Tel.: +1 (847) 471-8878, Fax: +1 (817) 258-8850 


\section{Abstract}

22 There is a need for more practical tools for estimating spore escape from crop canopies,

23 which is essential in forecasting the propagation of disease to other fields. In this paper,

24 we evaluated whether a random displacement model (RDM) parameterized with an

25 eddy diffusivity $K_{z}(z)$ could be used to predict spore escape probability. The proposed

26 RDM does not require detailed turbulence measurements for parameterization. Instead,

27 it constructs profiles of velocity and eddy diffusivity from a simple set of parameters

28 [canopy height, canopy density, vegetation length scale, and wind speed]. The RDM

29 was validated using field measurements of spore concentration. On average, the model

30 predictions matched the field measurements within $28 \%$ inside the canopy and $42 \%$

31 above it, comparable to LES results over the same canopy. Once validated, the RDM

32 was used to explore particle escape across a range of canopy densities and particle settling velocities, in order to inform estimates of particle escape from crops of varying

34 maturity or area density. Escape fraction as calculated by the RDM increased as canopy density decreased, as the ratio of particle settling velocity to turbulent shear

36 velocity ratio decreased, and as the source height within the canopy increased.

37 Keywords: particle transport; escape of particles from canopy; eddy diffusivity; random displacement model; maize 


\section{Introduction}

Fungicides are used in agricultural production to reduce losses due to fungal disease. Unfortunately, some fungicide components are transported into freshwater systems, causing sub-lethal effects on ecosystem processes, including fish reproduction and leaf decomposition (Elskus, 2012). Therefore, for both economic and ecologic reasons, it is desirable to reduce the amount of fungicides applied to crops, while maintaining their benefits to crop yield. Integrated Pest Management (IPM) reduces fungicide use by targeting applications to when the pathogen is most vulnerable (Roberts and Reigart, 2013). Because chemical treatments are most effective during the

51 first stages of fungal infection, it is necessary to forecast the spread of fungal spores to

52 effectively time fungicide applications (Aylor, 1999). Therefore, an understanding of

53 spore aerial transport is essential to the development of IPM strategies (Aylor and Irwin, 1999). For example, by correctly forecasting low soybean rust inoculum production and transport, the Integrated Aerobiology Monitoring System (IAMS) saved US soybean producers between $\$ 11$ and $\$ 229$ million in fungicide costs in 2005 (Isard et al., 2007). Evaluation of long distance spore transport must integrate the fungal life cycle, including spore release, escape from the canopy, transport and survival in the atmosphere, deposition on a new host, and infection of the host to generate new spores (Aylor 1986, 1990). This paper examines spore escape from the canopy, which depends on physical factors that determine the relative importance to spore escape of turbulent transport within the canopy, promoting escape, and spore settling, promoting deposition to the canopy and ground (e.g. Aylor and Taylor, 1983, Aylor, 1999, Aylor and Flesch, 2001, Nathan and Katul, 2005). 
The vertical structure of turbulence within a canopy is dependent on the canopy morphology, which is characterized by the canopy height $(h)$ and the plant frontal area

67 per unit canopy volume $\left(a_{f}\right)$. The dimensionless canopy density, or roughness density,

68

69

70

71

72

73

74

75

76

77

78 is defined as $a_{f} h$. If the canopy density is high $\left(a_{f} h \gtrsim 0.1\right)$, like most terrestrial crops, the drag imparted by the canopy is sufficient to generate an inflection point in the velocity profile, which leads to the generation of canopy-scale coherent structures at the top of the canopy through the Kelvin-Helmholtz instability (Raupach et al., 1996, Nepf, 2012). The canopy-scale vortices dominate the vertical turbulent transport of momentum and scalars, including spores, at the top of the canopy (Shaw et al., 1983; Ghisalberti and Nepf, 2005, 2006, Thomas and Foken, 2007). The region of the canopy flushed by these vortices is termed the exchange zone. The exchange zone extends from the top of the canopy over a distance $\delta_{e}=(0.23 \pm 0.06) /\left(C_{D} a_{f}\right)$, called the penetration length scale, with $C_{D}$ the canopy drag coefficient defined using a quadratic drag that includes the prefactor $1 / 2$ (Nepf et al., 2007).

Below the exchange zone exists a relatively quiescent region, termed the wake zone, within which turbulence is dominated by stem scale wakes, so that the vertical transport is greatly diminished relative to that in the exchange zone (Nepf et al., 2007). Because of the difference in vertical turbulent transport, spores originating in the wake zone, i.e., below the penetration of canopy-scale vortices, should have less likelihood of escaping the canopy than spores originating in the exchange zone, which can be flushed out by the canopy-scale vortices. Since the exchange zone decreases with increasing canopy density, the escape fraction should also decrease with increasing 
87 canopy density. The likelihood of particle escape also depends on the particle's size

88

89

90

91

92

93

94

95

96

97

98

99

100

101

102

103

104

105

106

107

108

109

and density, which dictate its settling velocity, $w_{s}$ (Aylor, 1990, Aylor, 1999).

Some researchers (e.g. Pan et al., 2014) have simulated particle transport in canopy flow using large eddy simulation (LES), which resolves large scales of turbulence, but represents the impact of small-scale turbulence using sub-grid scale parameterization. However, LES requires significant time and computational resources, precluding the investigation of a wide parameter space. For a less computationally intensive approach, researchers have proposed various forms of Lagrangian stochastic models (LSM), which produce ensembles of stochastic spore trajectories. Turbulence is represented through second or third-order turbulent correlations, which are typically parameterized using measured profiles of velocity variance, turbulent momentum flux, and TKE dissipation rate (Aylor, 1990, de Jong et al., 1991, Aylor and Flesch, 2001, de Jong, 1992, Andrade et al., 2009). In this paper we propose a random displacement model (RDM) that uses an eddy diffusivity to represent turbulent transport. The proposed RDM does not require the measurement of detailed velocity statistics or significant computational resources, so that it could be a valuable tool for driving models of long-range spore transport by providing rapid estimation of escape fraction from crops of varying maturity or area density. This approach assumes that turbulent motions are uncorrelated, so that the time averaged turbulent fluxes act as an enhanced Fickian diffusion, described by a turbulent diffusion coefficient $K_{z}$ (Legg and Powell, 1979, Aylor, 1982, Aylor, 1990, Aylor and Taylor, 1983, Denmead and Bradley, 1987). Because spores are released over hours, which is long compared to the duration of individual sweeps and ejections (6 to 10 s, Denmead and Bradley, 1987, Chamecki, 2013), an 
uncorrelated model should reasonably represent the time-averaged escape behavior.

111 Previous work has estimated eddy diffusivity from a canopy heat balance, or by using

112 second-order turbulence statistics to represent the time-mean eddy diffusivity, $K_{z}=$

$113 \sigma_{w}^{2} T_{L}$, with Lagrangian time scale $T_{L}=\frac{2 \sigma_{w}^{2}}{C_{0} \varepsilon}$, with $c_{0}$ a universal constant and $\varepsilon$ the rate of

114 turbulence dissipation (Durbin, 1983, Legg and Powell, 1979, Wilson and Sawford,

115 1996). However, these approaches require detailed, canopy-specific measurements of

116 turbulence statistics. In the proposed RDM, the velocity and eddy diffusivity profiles

117 within and above a canopy were constructed from existing equations for a neutral

118 boundary layer using a simple set of parameters [canopy height, canopy density,

119 vegetation length scale, and wind speed]. The RDM performance was evaluated

120 through a comparison to measured field data. After validation, the RDM was used to

121 explore the trends in escape fraction over a range of canopy densities $\left(C_{D} a_{f} h=0.1\right.$ to

1227 ), a range of settling velocities $w_{S} / u_{*}(0$ to 1$)$, and as a function of particle source

123 height, $z_{s r c} / h$ (0 to 1 , subscript 'src' denotes 'source').

124

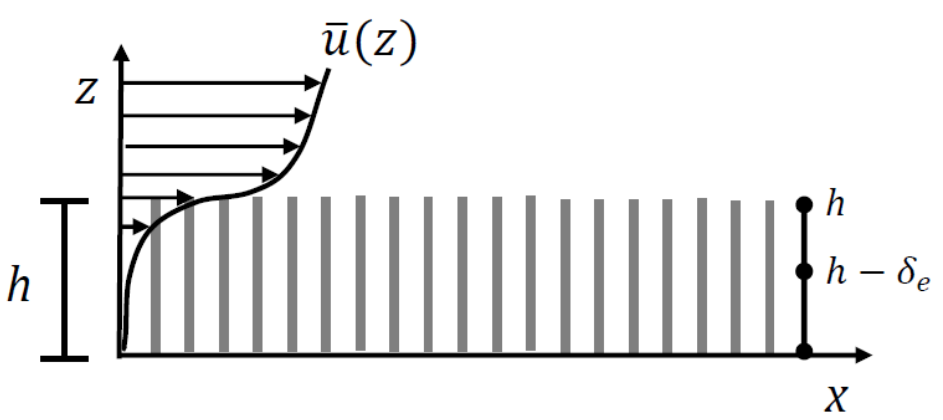

125

126

127

128

129
Figure 1 Schematic diagram of the lower part of the model domain, which extends to $z=10 \mathrm{~h}$. The longitudinal direction is $x$, the vertical direction is $z$, with $z=0$ at the ground, the canopy height is $h$, and the time-averaged longitudinal velocity is $\bar{u}(z)$. 


\section{Methods}

The RDM simulated a 2D domain (Figure 1) with the coordinates $x$ and $z$ parallel 132 and normal to the ground, respectively. The velocity vector $\vec{u}=(u, w)$ corresponded to 133 the streamwise and vertical coordinates $(x, z)$, respectively. The time average and 134 turbulent components of velocity were denoted by an overbar (e.g. $\bar{u}$ ) and prime (e.g. $135 u^{\prime}$ ), respectively. Individual particles originated at a specific source height, $z_{s r c}$, within 136 the canopy $\left(0<z_{s r c}<h\right)$ and were tracked until they deposited on the canopy, settled to 137 the ground, or left the modeling domain. Particles that reached $z / h=10$ were assumed 138 to have left the domain and were no longer tracked. The number of particles per run 139 (1000) was selected based on the fact that results with 1000 particles differed from 140 results with 10,000 particles by less than 5 percent. The size of the model domain $(x, z)$

141 was $18 h \times 10 h$. The sensitivity of results to domain size was evaluated by comparing 10 142 runs with a domain of $18 h \times 10 h$ to 10 runs with a domain of $200 h \times 10 h$. The difference 143 between escape fraction values determined in the two domains was less than $1442 \%$, indicating that the domain size did not significantly affect model results.

146 longitudinally with the mean velocity $\bar{u}$ and vertically due to both settling $\left(w_{s}\right)$ and

147 turbulent transport $\left(w^{\prime}\right)$. The equations used to model the particle position were (Wilson 148 and Sawford, 1996):

$$
x_{p, i+1}=x_{p, i}+\bar{u}\left(z_{p, i}\right) \Delta t
$$

$$
z_{p, i+1}=z_{p, i}+\left(\frac{d K_{z}\left(z_{p, i}\right)}{d z}-w_{s}\right) \Delta t+R \sqrt{2 K_{z}\left(z_{p, i}\right) \Delta t}
$$


151 The last term in (2) represents transport by turbulent velocity $w^{\prime}=R \sqrt{2 K_{z}} / \sqrt{\Delta t}$, and $R$ a 152 random number drawn from a normal distribution with mean 0 and standard deviation 1.

153 The vertical transport also included a drift correction, or pseudovelocity, associated with 154 the vertical variation in diffusivity $\left(d K_{z} / d z\right)$. The pseudovelocity term prevented the 155 artificial accumulation of particles in regions of low diffusivity (Durbin, 1983, Boughton et 156 al., 1987, Wilson and Sawford, 1996, Wilson and Yee, 2007). The formulations for the 157 vertical profiles of time-mean streamwise velocity, $\bar{u}(z)$, and eddy diffusivity, $K_{z}(z)$ are 158 described below in Section 2.1. The particle position was saved at every time-step. The 159 model time-step, $\Delta t$, was constrained so that the vertical particle excursion within each 160 time-step was much smaller than the scale of vertical gradients in the diffusivity and 161 velocity (Israelsson et al., 2006). Within the model canopy, both the velocity and 162 diffusivity varied over length scales of approximately $0.1 \mathrm{~h}$. For each run the time-step 163 satisfied the following condition:

164

$$
\Delta t<\min \left(\frac{0.1 h}{\left.\left|\frac{\partial K_{Z}}{\partial z}-w_{S}\right|\right|_{\max }}, \frac{(0.1 h)^{2}}{\left.K_{z}\right|_{\max }}\right) .
$$

Within each time-step, after a particle was moved, the position was assessed to 166 determine if the particle had settled to the ground $\left(z_{p, i}=0\right)$; escaped the canopy $167\left(z_{p, i}>h\right)$; escaped the model domain $\left(z_{p, i}>10 h\right.$ or $\left.x_{p, i}>18 h\right)$; or deposited to the 168 canopy. For a continuous release, as considered here, the fraction of spores escaping 169 the canopy is a function of distance from the source, but not time. As described in Pan 170 et al. (2014), the escape fraction initially increases with distance from the source, 171 reaching a maximum at $x / h=2$ to 6 , depending on settling velocity (Figure 7 in Pan et 
172 al., 2014). Since the canopy is a sink for particles, over longer distances particles may

173 return to the canopy through turbulent transport or settling and be deposited, so that at

174 larger distances the escape fraction exhibits a slow decline with distance. To provide a 175 single, consistent metric with which to compare different scenarios in Section 3.2, we 176 adopt the escape fraction metric $(E F)$ defined by Pan et al. (2014) as the maximum 177 fraction of the released particles observed above the canopy.

Canopy deposition was described using a modified version of the model given in 179 Aylor and Flesch (2001). Deposition on vertical facing and upward facing surfaces was possible if the velocity was less than the critical velocity $u_{\text {crit }}=0.45 \mathrm{~m} / \mathrm{s}$, determined by Aylor (2005) for pollen capture in a maize canopy. Particle rebound and re-

182 entrainment was expected if the particle velocity was greater than $u_{\text {crit }}$. We caution that 183 the value of $u_{\text {crit }}$ may vary with particle type (pollen versus spores), and also with 184 canopy rigidity and morphology. Following Pan et al. (2014), deposition on downward 185 facing surfaces was neglected. Deposition on upward facing surfaces was possible if 186 the particle had a negative vertical particle excursion, $z_{p, i+1}-z_{p, i}<0$. The rate of 187 deposition on an upward facing surface $\left(S_{u}\right)$ was calculated as the product of the two188 sided leaf area density, $a(z)$, the fractional projected leaf area normal to the vertical 189 direction $\left(P_{z}\right)$, and the settling velocity:

$$
S_{u}=P_{z} a(z) w_{s}
$$

191 Pan et al. (2014, eq. A2) described particle deposition to vertical surfaces in a three192 dimensional domain. Here, we modified the formulation for the two-dimensional domain $193(x-z)$ of this RDM. Specifically, the projected area in the $x$ direction was assumed to be 
194 the sum of the measured projected leaf area facing the $x\left(P_{x}\right)$ and $y\left(P_{y}\right)$ directions in 3D 195 space $\left(P_{x, 2 D}=P_{x}+P_{y}\right)$, and the rate of impaction depended only on the mean 196 longitudinal velocity $(\bar{u})$ since $\bar{v}=0$ in the 2D domain. The rate of deposition on 197 vertical surfaces $\left(S_{v}\right)$ is then given by:

$$
S_{v}=E I\left(P_{x, 2 D}\right) a(z) \bar{u}
$$

with impaction efficiency $(E I)$,

$$
E I=0.86\left(1+0.442 S t^{-1.967}\right)^{-1}
$$

201

202

203

204

205

206

207

208

209

210

211

212

213

214

based on Aylor (1982). In eq. (6), St is the Stokes number $S t=\frac{w_{S} \bar{u}}{g L_{v}}$, with $L_{v}$ the characteristic length scale of the canopy elements and $g$ the gravitational acceleration. Because the RDM tracked individual particles, the time rate of deposition given by (4) through (6) was converted to a probability for individual particle deposition during one time-step. Following Aylor (1989), the rate of deposition, $S_{u}+S_{v}$, was multiplied by $\Delta t$, resulting in a number between 0 and 1 that represented the probability of deposition during that time-step. The need to keep the fraction of particles deposited in each timestep less than 1 imposed an additional constraint on the time-step; however, this condition was satisfied by the more stringent constraints on the vertical particle excursion (eq. 3). To determine if the particle deposited during the time-step, a random number, $R_{c}$, was chosen from a uniform distribution between 0 and 1. If $R_{c}$ was less than or equal to the probability of deposition, the particle deposited to the canopy.

\subsection{Velocity and Eddy Diffusivity Profiles to Parameterize RDM}


217 canopy, $u_{*}{ }^{4}=\left(\overline{u^{\prime} w^{\prime}}\right)_{h}^{2}+\left(\overline{v^{\prime} w^{\prime}}\right)_{h}^{2}$ (In this paper, the velocity field is assumed to be 218 aligned with $x$ and uniform in $y$, so that $\overline{v^{\prime} w^{\prime}}=0$ ). Profiles were only constructed for 219 dense canopies $\left(a_{f} h \gtrsim 0.1\right)$, representative of most terrestrial crops. Within the canopy 220 the profiles are divided into two regions. In the wake zone $\left(z<h-\delta_{e}\right)$, both $K_{z}$ and $\bar{u}$ 221 are small, so particle transport is likely dominated by settling $\left(w_{s}\right)$. In the exchange zone $222\left(z \geq h-\delta_{e}\right)$, canopy-scale vortices elevate $K_{z}$ and contribute to greater momentum 223 penetration from above, and thus higher $\bar{u}$, so that both turbulent transport and capture 224 to the canopy may become important processes for spore transport. canopy, there is a displaced boundary-layer profile:

$$
\bar{u}(z)=\frac{u_{*}}{\kappa} \ln \left(\frac{z-z_{m}}{z_{0}}\right)
$$

228 in which $\kappa=0.4$ is the von Kármán constant (Raupach, 1994, Thom, 1971). The 229 displacement height $\left(z_{m}\right)$ and roughness height $\left(z_{0}\right)$ are both functions of canopy 230 density (e.g., Schlichting 1936, Grimmond and Oke, 1999). Following Luhar et al. 231 (2008), the displacement height $z_{m}$ and roughness height $z_{o}$ can be described in terms 232 of $C_{D} a_{f} h$,

$$
\frac{z_{m}}{h}=1-\frac{0.12}{C_{D} a_{f} h}
$$

$$
\frac{z_{o}}{h}=0.04\left(C_{D} a_{f} h\right)^{-1} \quad C_{D} a_{f} h \geq 0.1
$$


235 The velocity inside the canopy follows an exponential decay, e.g. combining Harman and Finnigan (2007) and Nepf (2012):

$$
\bar{u}(z)=u_{1}+\left(u_{h}-u_{1}\right) e^{\frac{\beta(z-h)}{l}}
$$

238 in which $u_{h}$ is the velocity at the top of the canopy, and $u_{1}$ is the velocity in the lower 239 canopy (wake zone), below the penetration of vertical turbulent momentum flux. The 240 ratio $u_{1} / u_{h}$ decreases with increasing canopy density, and the following relation was 241 determined by fitting data from terrestrial canopies reported in Figure 1 and Table 1 in 242 Finnigan (2000):

$$
u_{1} / u_{h}=0.16\left(a_{f} h\right)^{-0.68}
$$

Given $u_{*}$, equations (7) to (9) predict the velocity at the top of the canopy, $u_{h}=u(h)$. Specifically, $u_{h} / u_{*}=2.7$ for all values of $a_{f}$, which is consistent with observations made across a wide range of dense aquatic and terrestrial canopies (Ghisalberti, 2009). The

247 velocity-decay length-scale $(l / \beta$ in 10$)$ can be determined using a mixing length $(l)$

248 characterization of eddy viscosity, which leads to $\beta=\frac{u_{*}}{u_{h}}$ and $l=\frac{2 \beta^{3}}{C_{D} a_{f}}$ (Harman and 249 Finnigan, 2007).

250 Next, consider the vertical profile of eddy diffusivity. Previous parameterizations of 251 eddy diffusivity, such as Massman and Weil (1999), do not reflect the contributions of 252 the coherent structures at the top of the canopy, or the role of the plant-scale vortices 253 within the canopy, both of which have been recently shown to provide important controls 254 on the magnitude of diffusivity within the canopy (Poggi et al., 2004, Tanino and Nepf, 255 2008). The models used here incorporate both of these important length-scales. In the 
256 wake zone $\left(z<h-\delta_{e}\right)$, the diffusivity is dominated by plant-scale turbulence, and $K_{z}$

257 scales on the characteristic vegetation length scale $\left(L_{v}\right)$ and the velocity scale $\sqrt{k_{t}}$

258 associated with the turbulent kinetic energy $\left(k_{t}\right)$ generated in the plant wakes (Raupach

259 et al., 1996, Finnigan, 2000, Tanino and Nepf, 2008). Tanino and Nepf (2008)

260 developed models for $k_{t}$ and $K_{z}$ as functions of canopy morphology $\left(L_{v}\right.$ and $\left.a_{f}\right)$ and

261 local velocity $(\bar{u})$. Most crops have a low solid volume fraction $\left(\phi=a_{f} t\right.$, with $t$ the blade

262 thickness), such that plant-scale eddies can exist throughout the canopy and have scale

$263 L_{v}$, so that equations (2.12) and (2.15) in Tanino and Nepf (2008) reduce to:

$264 \quad \frac{K_{z}}{\bar{u} L_{v}}=4.5\left(\frac{\sqrt{k_{t}}}{\bar{u}}\right)$

$265 \quad \frac{\sqrt{k_{t}}}{\bar{u}}=1.1\left(C_{D} \frac{a_{f} L_{v}}{2}\right)^{1 / 3}$

266 in which the scale coefficients (1.1 and 4.5) were determined in laboratory experiments 267 (Tanino and Nepf, 2008).

268 In the upper canopy $\left(h-\delta_{e}<z<h\right)$, the flow resembles a mixing layer (Raupach et 269 al., 1996), within which the eddy diffusivity follows a mixing length model (e.g. Poggi et 270 al., 2004),

$271 \quad K_{z}(z)=\left(\frac{1}{s c}\right) l_{e f f}^{2} \partial \bar{u} / \partial z$

272 with effective eddy length-scale $l_{e f f}$. The turbulent Schmidt number, $S c$, was assumed 273 to be equal to 0.5 , as in a mixing layer (Raupach et al.,1996). This is consistent with 274 recent laboratory experiments for which $S c$ equal to 0.47 was measured within and 275 above a model canopy in a neutrally stable flow (Ghisalberti and Nepf, 2005). A range 
276 of $S c$ values between 0.5 and 1 has been observed above crop canopies, with $S c$

277 increasing with atmospheric stability and dependent on scalar species (Wilson, 2013).

$\partial u /\left.\partial z\right|_{z=h}=u_{h} / \delta_{e}$ in (14). Thus, the diffusivity at the top of the canopy $(z=h)$ is

$281 K_{h}=2 l_{e f f}{ }^{2} u_{h} / \delta_{e}$, which is the local maximum in diffusivity. For simplicity, a linear

282 transition was assumed between $K_{h}$ at $z=h$ and the lower canopy value given by eq.

283 (12) at $z=h-\delta_{e}$. The contribution of the mixing-layer vortices to $K_{z}$ was assumed to be scale, $\delta_{e}$, above the canopy. of the mixing-layer $\left(l_{M L}\right)$ and boundary-layer $\left(l_{B L}\right)$ length scales:

$$
l_{e f f}=(1-\alpha) l_{B L}+\alpha l_{M L}
$$

$$
l_{B L}=\kappa\left(z-z_{m}\right)
$$

291 The relative contribution of the mixing layer length scale $(\alpha)$ is a function of $C_{D} a_{f} h$.

292 Figures 8 and 9 from Poggi et al. (2004) showed that $\alpha$ reached an asymptote of

$293 \alpha=0.45$ for $C_{D} a_{f} h \geq 0.6$, and $\alpha=0.25$ to 0.45 over the range $C_{D} a_{f} h=0.1-0.6$. Using

$294 u_{h} / u_{*}=2.7$ (determined above), $\beta=u_{*} / u_{h}=0.37$, which leads to

$295 l_{M L}=0.27\left(C_{D} a_{f}\right)^{-1} \approx \delta_{e}$; i.e., the eddy length scale in the mixing layer corresponds to 
296 the length scale of turbulence penetration into the canopy. Note that as $C_{D} a_{f}$

297 approaches zero, (17) implies that $l_{M L}$ is unbounded, which is not physically reasonable.

298 To correct this, $l_{M L}$ is constrained to be the minimum of eq. (17) and $h$. Finally, above

$299 z=h+\delta_{e}$, the eddy diffusivity follows the boundary-layer form,

300

$$
K_{z}(z)=\left(\frac{1}{s c}\right) \kappa u_{*}\left(z-z_{m}\right) \quad z>h+\delta_{e},
$$

301 with $S c=0.8$ for a boundary layer (Launder, 1976, Hassid, 1983, Koeltzch, 2000).
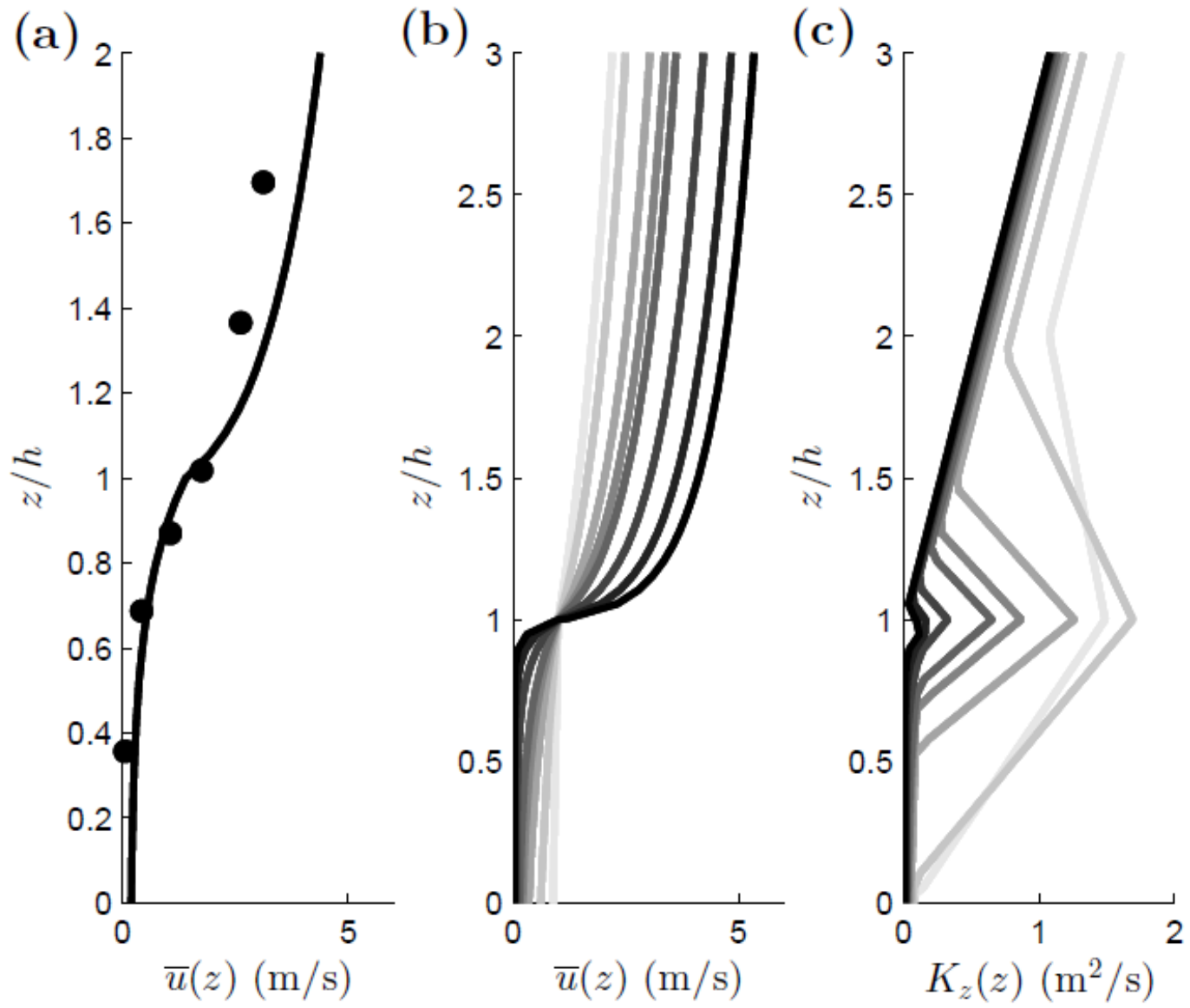

303 Figure 2 (a) time-averaged velocity $\bar{u}(\mathrm{z})(\mathrm{m} / \mathrm{s})$ predicted from eqs. (7) to (10) shown by solid line and measured over a maize field (Gleicher et al., 2014), shown by dots. Family of curves describing (b) time-averaged streamwise velocity $\bar{u}(\mathrm{z})(\mathrm{m} / \mathrm{s})$ and (c) vertical turbulent diffusivity $K_{z}(z)\left(\mathrm{m}^{2} / \mathrm{s}\right)$ predicted from eqs. (7) to (18). From light to dark, curves represent increasing canopy density, with $C_{D} a_{f} h=0.1,0.25,0.5,0.75,1,2,4$, and 7 , respectively, with $u_{*}=0.51 \mathrm{~m} / \mathrm{s}$, 
$h=2.1 \mathrm{~m}, L_{v}=0.1 \mathrm{~m}$, and $C_{D}=0.68$ for all curves, based on a maize canopy, as described in the text.

Figure 2a shows velocity measured in a maize field (dots) and the time-averaged velocity constructed from eqs. (7) through (10) using parameters from that maize field, specifically $u_{*}=0.51 \mathrm{~m} \mathrm{~s}^{-1}, h=2.1 \mathrm{~m}$, and $L A I=3.3$. (Gleicher et al., 2014).

Following Finnigan (2000), the frontal area density was assumed to equal $1 \frac{1}{2}$ the onesided leaf area index, $a_{f} h=\frac{1}{2} L A I$. Wilson et al. (1982) measured $C_{D}=0.17$ for maize, but used a drag formulation that excludes the factor of $\frac{1}{2}$, which we include, and used the single-sided leaf area, whereas we use $\frac{1}{2} L A I$. To compensate, we adjusted $C_{D}$ by a factor of 4 , such that $C_{D}=0.68$. Using only the reported values of $u_{*}, h, C_{D}$, and $L A I$ the constructed velocity profile (solid line in Figure 2a) agreed with the measured velocity (dots in Figure 2a) to within $0.26 \mathrm{~m} / \mathrm{s}$ inside the canopy $(z / h \leq 1)$, and to within $0.85 \mathrm{~m} / \mathrm{s}$ above the canopy $(1<z / h \leq 1.7)$.

Figures $2 b$ and $2 c$ show the family of curves constructed from eqs. (7) through (18) using $C_{D} a_{f} h=0.1$ to 7 . The velocity profiles resemble the family of measured velocity profiles shown in Figure 1 of Finnigan (2000). Recall that only dense canopies have been considered $\left(C_{D} a_{f} h>0.1\right)$, and consistent with this each velocity profile resembles a mixing layer, with a velocity inflection point at the top of the canopy. Importantly, the model captures the peak in diffusivity at the top of the canopy (Figure 2c) associated with the coherent structures formed in the mixing-layer, a feature that is not captured by the model proposed by Massman and Weil (1999). At the lowest densities considered (lightest lines), the coherent structures penetrate to the ground $\left(\delta_{e}=h\right)$, elevating diffusivity over the full canopy. As canopy density increases, the 
331 exchange zone decreases in size $\left(\delta_{e} \sim\left(C_{D} a_{f}\right)^{-1}\right)$.The mixing layer eddy length-scale,

$332 l_{M L} \sim\left(C_{D} a_{f}\right)^{-1}$, also decreases, leading to a diminished diffusivity at the top of the

333 canopy.

\begin{tabular}{cccc} 
Figure & $w_{s} / u_{*}$ & $a_{f} h$ & $h(\mathrm{~m})$ \\
\hline $2 \mathrm{a}$ & - & 1.65 & 2.1 \\
$2 \mathrm{~b}, \mathrm{c}$ & - & $0.15-10.29$ & 2.1 \\
3 & 0.04 & 1.65 & 2.1 \\
4 & $0.01-1$ & 1.65 & 2.1 \\
$5 \mathrm{a}-\mathrm{d}$ & $0-5$ & $0.15-10.29$ & 2.1 \\
$5 \mathrm{e}, \mathrm{f}$ & $0,0.1$ & $0.15-10.29$ & $0.067-4.67$ \\
Gleicher et al. $(2014)$ & 0.04 & 1.65 & 2.1 \\
\hline$C_{D}=0.68, u_{*}=0.51 \mathrm{~m} / \mathrm{s}, L_{v}=0.1 \mathrm{~m}$ in all figures
\end{tabular}

334

335 Table 1 Description of parameters used for each set of simulations

336

337

3. Results and Discussion

338

\subsection{Validation using field data from maize canopy}

339

The RDM was validated against measurements from a field release of

340

Lycopodium spores $\left(w_{s}=1.94 \mathrm{cms}^{-1}\right)$ in a maize canopy (Gleicher et al., 2014), which

341 is the same study used for comparison in Figure 2a. Gleicher et al. (2014) report

$342 u_{*}=0.51 \mathrm{~m} \mathrm{~s}^{-1}, h=2.1 \mathrm{~m}, L A I=3.3$. The characteristic vegetation length scale is the

343 leaf width, $L_{v}=0.1 \mathrm{~m}$ (Silva et al., 2012). Gleicher released spores from a single pole

344 at three source heights $\left(\frac{z_{s r c}}{h}=1, \frac{2}{3}, \frac{1}{3}\right)$ and captured spores using a grid of 9 poles, with 5

345 rotorods per pole $\left(\frac{x}{h}=0.94,1.9,3.8, \frac{y}{h}=-0.36,0,0.36, \frac{z}{h}=0.34,0.68,1.0,1.4,1.7, h=2.1\right.$

$346 \mathrm{~m})$. Rotorod data was missing from three locations $\left(\frac{x}{h}=1.9,3.8, \frac{y}{h}=0.36\right)$, so data for 
347 these three rotorod locations was taken from the corresponding rotorods at the opposite 348 poles $\left(\frac{x}{h}=1.9,3.8, \frac{y}{h}=-0.36\right)$.

We calculated concentration and airborne flux in the RDM using a method similar to Gleicher et al. (2014). A vertical column of interrogation boxes $(0.2 \mathrm{~m}$ long $\times 0.1 \mathrm{~m}$ high) was defined, centered at the field data collection points. The particle concentration was found by dividing the number of airborne particles in each box by the box area. Particles were continuously released until a steady-state particle concentration was established in each box. Because RDM is two dimensional in $x-z$, it cannot represent the lateral dispersion present in the field. Therefore, the field data was adjusted to correct for the fraction of particles lost from the control volume by lateral dispersion. Specifically, the field data was normalized by the equivalent two-dimensional flux 358 represented within the measurement volume, $Q_{x}=\int_{-0.76 m}^{0.76 m} \int_{0.7 m}^{3.5 m} \bar{u} C d z d y$ ), with the 359 integral approximated by trapezoidal summation. A laterally-integrated concentration was found from the measurements at the three $y$ locations $\left(\langle C\rangle=\int_{-0.76 m}^{0.76 m} C(x, y, z) d y\right)$, 361 which has units of spores $\mathrm{m}^{-2}$. The concentration within the 2-D RDM was, by definition, 362 the laterally-integrated value, $\langle C\rangle$. The RDM concentration was scaled by the two363 dimensional flux at each $x$ location $\left(Q_{x}=\int_{0.7 m}^{3.5 m} \bar{u}\langle C\rangle d z\right.$, which has units of spores $\left.\mathrm{s}^{-1}\right)$, 364 with the integral approximated by trapezoidal sums. Using this method, both the RDM 365 and field normalized flux profiles $\frac{\bar{u}\langle C\rangle}{Q_{x}}$ integrate to 1. The field measurement $\left(C_{f}\right)$ and predicted $\left(C_{p}\right)$ spore concentrations are shown in 367 Figure 3. With $\bar{u}(z)$ and $K_{z}(z)$ predicted from eqs. (7) to (18) using the measured 
368

369

370

371

372

373

374

375

376 377 Figure 2a).

canopy parameters from Gleicher (summarized in Table 1), $73 \%$ of the predicted spore concentrations were within a factor of 2 of the field observations $(F A C 2=73 \%$; fraction of data that satisfy $0.5 \leq \frac{C_{p}}{C_{f}} \leq 2.0$ ) (Chang and Hanna, 2008). The agreement improved with distance from the source (Figure 3g,h,i; Table 2), which is consistent with the expectation that the RDM makes better predictions in the far-field. At the farthest measurement point $(x / h=3.8)$, and for all source heights, the prediction concentrations were, on average, 1.28 times the field measurements within the canopy and 0.58 times the field measurements above it. The underprediction of concentration above the canopy may have been related to the overprediction of $\bar{u}$ in this region (see

For additional comparisons, the geometric mean bias $(V G)$ and ratio of geometric means, or mean geometric bias $(M G)$ were also assessed (Hanna et al., 1993, Chang and Hanna, 2004):

$$
\begin{aligned}
& V G=e^{\left(\overline{l n} \frac{\overline{C_{f}}}{C_{p}}\right)^{2}} \\
& M G=e^{\sqrt{\overline{n C_{f}}}-\sqrt{n C_{p}}}
\end{aligned}
$$

in which the tilde indicates the spatial average over all rotorod heights. Chang and Hanna (2004) suggest that a "good" model should have greater than $50 \%$ of model predictions within a factor of 2 of observations ( $F A C 2>50 \%$ ), a geometric mean bias $(V G)$ less than 1.6, and a ratio of geometric means within $30 \%(0.7<M G<1.3)$. The $F A C 2, V G$, and $M G$ values for each measurement location and release height are shown in Table 2. All statistics improved with $x / h$, approaching or exceeding the 
389

390

391

392

393

recommendation from Chang and Hanna (2004). The geometric mean bias, which reflected the ratio between $C_{f}$ and $C_{p}$ on a logarithmic scale, was unacceptably large for $x / h=0.95,1.9$, suggesting an initially lower dispersion of the RDM relative to the field measurements. By $x / h=3.8$, all statistics were within the range of a "good" model (Chang and Hanna, 2004).

Recently, an LES model (Pan et al, 2014) and a first-order LSM with memory terms (Gleicher et al., 2014) were compared to the same field release of Lycopodium spores. The performance of all three models is compared in Table 2. At $x / h=3.8$, the RDM performed similarly to the LES and better than the LSM. Note that the LES performance declined with source height (Table 2), and that the RDM performed better for $z_{s r c} / h=2 / 3$ and $1 / 3$. This is because the RDM included the contribution of plantscale eddies in the lower canopy (see eq. 12 and 13). The LES model used a distributed drag to represent the canopy, which did not produce leaf-scale eddies and their contribution to mass flux in the lower canopy, such that the LES underestimated the diffusivity in the lower canopy. Consistent with this, below the penetration of canopy scale vortices $\left(z \leq h-\delta_{e}\right)$, the far field eddy diffusivity (derived by dividing the LES local mean vertical flux by the vertical gradient in mean concentration) was 6 times smaller than the eddy diffusivity predicted by eqs. (12) and (13), which was used in the RDM. Because the region below the penetration of canopy scale vortices comprises a significant fraction of dense canopies, it is important to correctly represent vertical transport in this region. The performance of the LES in the lower canopy might be improved by incorporating eq. (13) to represent the contribution of unresolved, plantscale eddies. 
Because the RDM does not resolve individual sweeps and ejections, it can only

413 model far field particle behavior, after particles have been in transport for more than 10

414 Lagrangian timescales $\left(\tau_{l} \cong 0.28-0.63 \mathrm{~s}\right.$, based on velocity measurements of the

415 Eulerian integral time scale made during the same maize field study, Chamecki, 2013).

416 To explore at what point far field conditions began in the RDM, the transport time for

417 airborne particles to reach several $x$ locations was tracked. The far-field condition was

418 reached at $x / h=5$, at which point over $95 \%$ of the airborne particles had been in

419 transport for more than 10 Lagrangian timescales. Because the maximum escape in the

420 RDM occurred beyond this point, an eddy diffusivity-based approach should adequately

421 represent $E F$. This, together with the agreement between the modeled and measured

422 spore concentrations, built confidence in the RDM model, which was next used to

423 evaluate trends in $E F$ with particle size $\left(w_{s} / u_{*}\right)$ and canopy density $\left(C_{D} a_{f} h\right)$.

\begin{tabular}{|c|cccccc|}
\hline$z_{\text {src }} / h$ & statistic & $x / h=0.95$ & $x / h=1.9$ & $x / h=3.8$ & LES & LSM \\
\hline \multirow{2}{*}{1} & $F A C 2$ & $80 \%$ & $80 \%$ & $80 \%$ & $93 \%$ & $55 \%$ \\
& $M G$ & 1.52 & 1.43 & 1.17 & 1.02 & 1.87 \\
& $V G$ & 1.79 & 1.27 & 1.20 & 1.17 & 2.05 \\
\hline \multirow{2}{*}{$2 / 3$} & $F A C 2$ & $60 \%$ & $80 \%$ & $80 \%$ & $70 \%$ & $67 \%$ \\
& $M G$ & 2.07 & 1.56 & 1.09 & 0.70 & 1.13 \\
& $V G$ & 19.5 & 2.18 & 1.37 & 1.42 & 1.80 \\
\hline \multirow{2}{*}{$1 / 3$} & $F A C 2$ & $40 \%$ & $80 \%$ & $80 \%$ & $66 \%$ & $71 \%$ \\
& $M G$ & 2.78 & 1.51 & 1.09 & 1.09 & 1.02 \\
& $V G$ & 153 & 2.50 & 1.39 & 2.18 & 1.45 \\
\hline
\end{tabular}

Table 2 Fraction of RDM predictions within a factor of 2 of field observations (Gleicher et al., 2014) (FAC2), ratio of geometric means (MG), and geometric mean bias (VG) at three $x / h$ locations and three source heights. Quantitative statistics representing the average value of the comparison to field data at all longitudinal locations $(x / h=$ 
$4290.95,1.9,3.8$ ) are included for an LES model (Pan et al., 2014) and a first order 430 Lagrangian stochastic model (Gleicher et al., 2014)
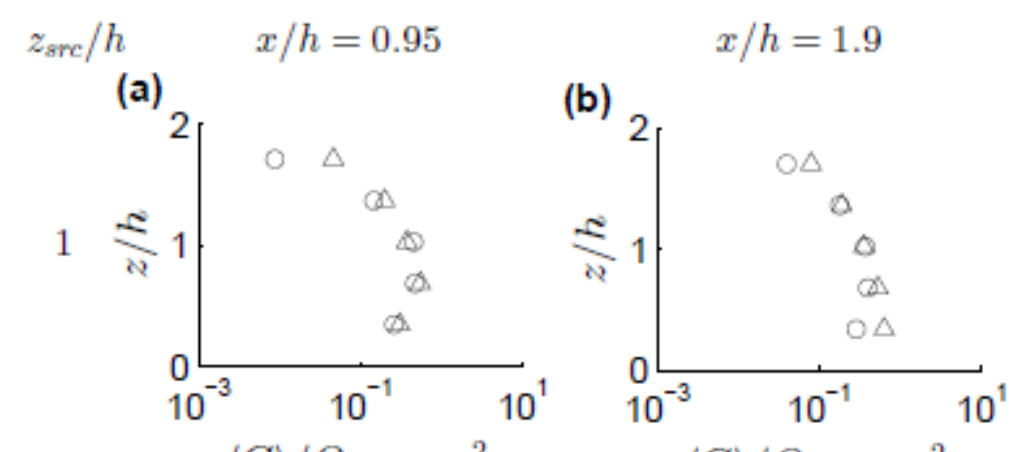

$x / h=3.8$
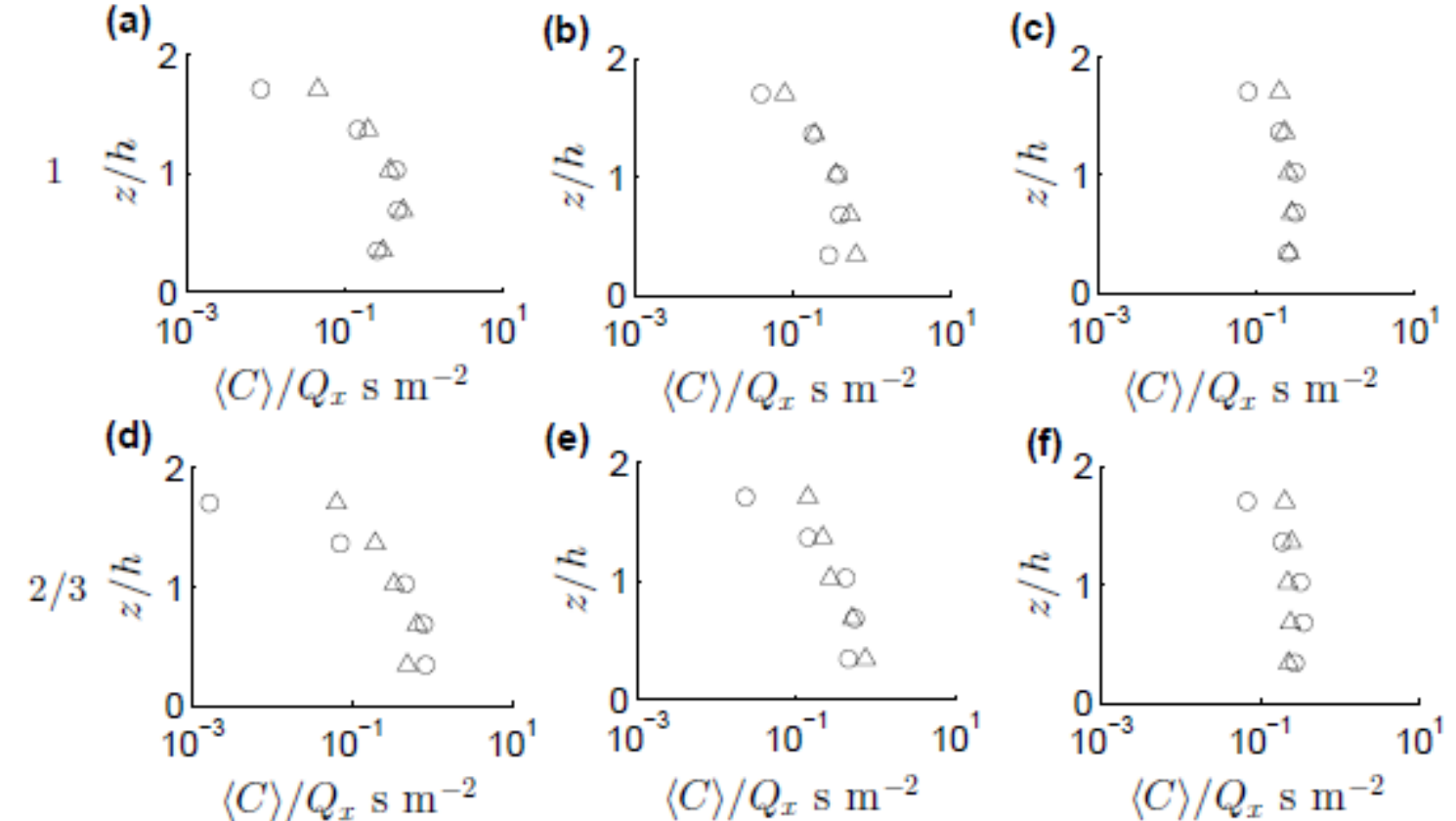

$\langle C\rangle / Q_{x} \mathrm{~s} \mathrm{~m}^{-2}$

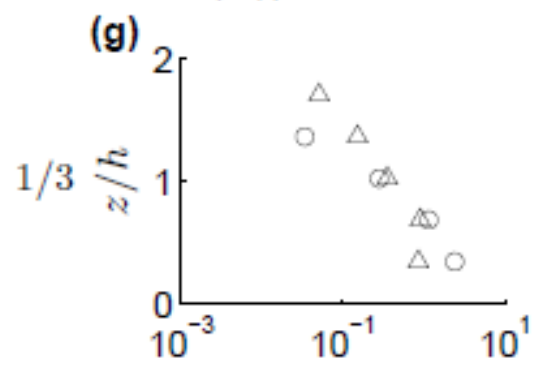

$$
\langle C\rangle / Q_{x} \mathrm{~s} \mathrm{~m}^{-2}
$$
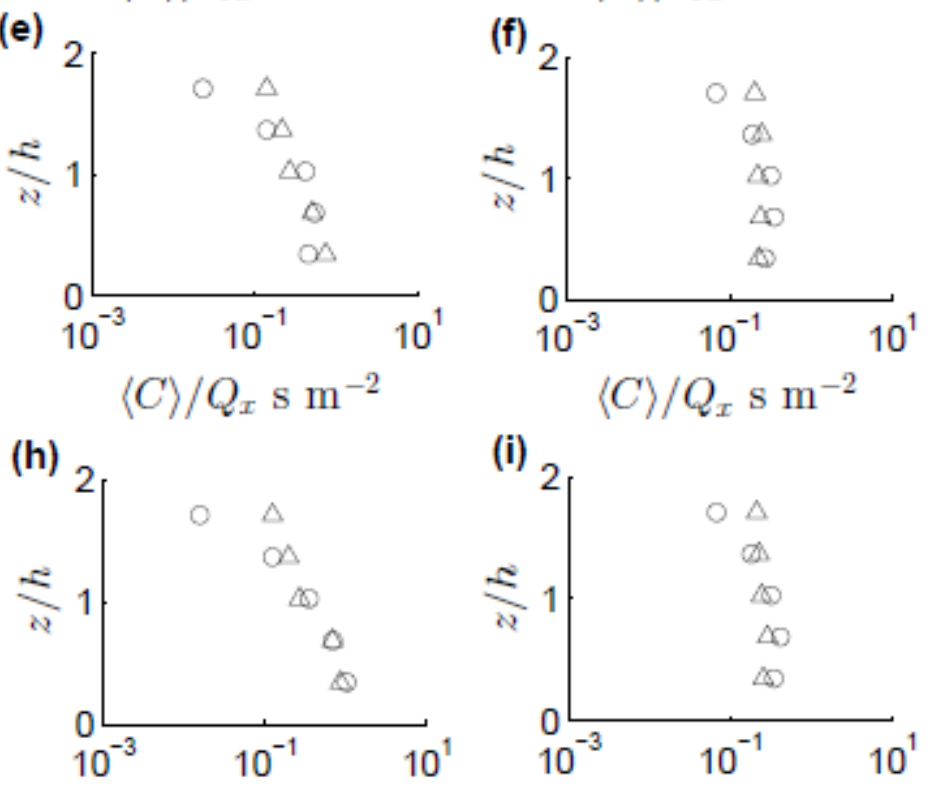

$$
\langle C\rangle / Q_{x} \mathrm{~s} \mathrm{~m}^{-2}
$$

$\langle C\rangle / Q_{x} \mathrm{~s} \mathrm{~m}^{-2}$

432 Figure 3 Comparison of the concentration predicted by RDM-maize (open circles) with measured concentration of Lycopodium spores released in a maize canopy (Gleicher et al., 2014), shown with open triangles. The laterally integrated concentration $\langle C\rangle$ is normalized by the equivalent source flux $\left(Q_{x}\right)$, and vertical coordinate $(z)$ is normalized by canopy height $h$. Spores were released at $z_{s r c} / h=1 / 3(\mathrm{a}, \mathrm{b}, \mathrm{c}), 2 / 3(\mathrm{~d}, \mathrm{e}, \mathrm{f})$, and 1 $(\mathrm{g}, \mathrm{h}, \mathrm{i})$. The spores were collected at $x / h=0.95(\mathrm{a}, \mathrm{d}, \mathrm{g}), 1.9(\mathrm{~b}, \mathrm{e}, \mathrm{h}), 3.8(\mathrm{c}, \mathrm{f}, \mathrm{i})$. 


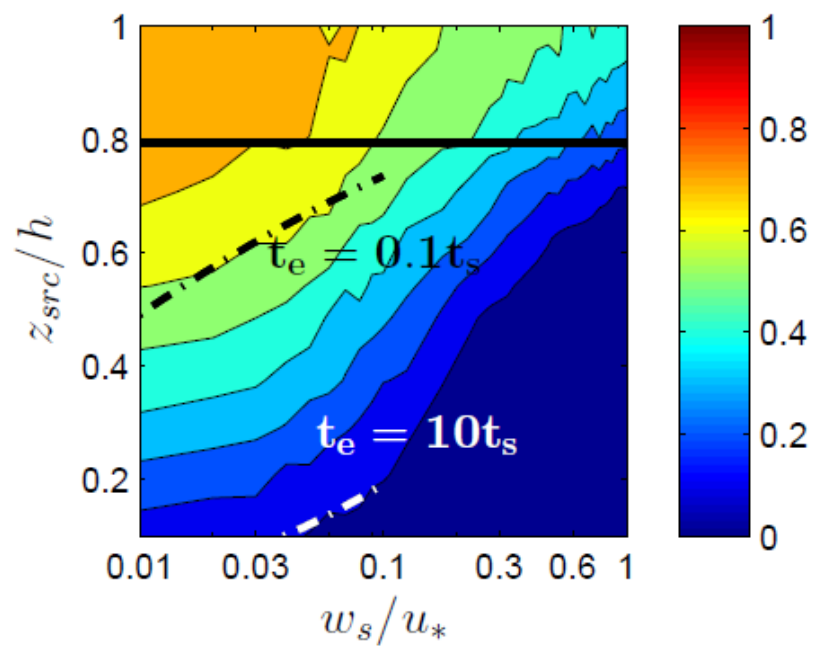

Figure 4 Particle escape fraction ( $E F$, values shown in color bar) from a maize canopy $(L A I=$ 3.3) as a function of the source height, $z_{s r c}$, normalized by canopy height $h$, and particle settling velocity $w_{s}$, normalized by friction velocity $u_{*}$. The solid black horizontal line denotes the depth of turbulence penetration from above $\left(z=h-\delta_{e}\right)$. Lines comparing the time-scale of escape $\left(t_{e}\right.$, eq. 20) and of settling ( $t_{s}$, eq. 21) are also shown. The lines represent the locus in $\left(z_{s r c} / h, w_{s} / u_{*}\right)$ space for which $t_{e}=\gamma t_{s}$ with $\gamma=0.1$ (black dashed line) or 10 (white dashed line). The solid black contour lines separating regions of different color represent successively the $E F$ contours 0.1 to 1 in intervals of 0.1 .

RDM was used to explore how the velocity ratio $\left(w_{s} / u_{*}\right)$, canopy density, and canopy height impacted escape fraction, an investigation of 1788 simulations (Figures was used to explore how the velocity ratio impacted escape fraction (Figure 4, model parameters given in Table 1). The solid horizontal line indicates the expected

454 penetration of shear-layer turbulence from above the canopy, i.e. $z=h-\delta_{e}$. Consider 455 particles with settling velocity comparable to the turbulent velocity $\left(w_{s} / u_{*}=1\right)$. These 456 particles only escaped if they originated in the exchange zone $\left(z_{s r c}>h-\delta_{e}\right)$, i.e. the 457 region within which turbulent transport is enhanced by the canopy-scale vortices formed 
458 at the top of the canopy. Escape from the wake zone $\left(z_{s r c}<h-\delta_{e}\right)$ was unlikely

$459(<10 \%)$, because of the significantly lower $K_{z}$ associated with the stem-scale vortices

460 that dominate transport in this region (eq. 12). In contrast, particles with relatively small

461 settling velocity $\left(w_{s} / u_{*}<0.1\right)$ could be moved significant distances by the lower canopy

462 turbulence, allowing escape even for particles originating deep within the canopy. For

463 these light particles $\left(w_{s} / u_{*}<0.1\right)$, canopy deposition was less important (impacting less

464 than $20 \%$ of the particles, data not shown), so that the trends in escape fraction could

465 be predicted by comparing the time scale for turbulent transport to the top of the canopy

$466\left(t_{e}\right)$ and the time scale for settling to the ground $\left(t_{s}\right)$ :

$467 \quad t_{e}=\frac{\left(h-z_{s r c}\right)^{2}}{K_{z}\left(z_{s r c}\right)}$

468

$$
t_{s}=\frac{z_{s r c}}{w_{s}}
$$

469 Figure 4 depicts the locus in $\left(z_{s r c} / h, w_{s} / u_{*}\right)$ space for which $t_{e}=\gamma t_{s}$ with $\gamma=0.1$ (black

470 dashed line) and 10 (white dashed line). Escape was rare $(<10 \%)$ if $t_{e} / t_{s}>>1$,

471 corresponding to particles originating below the lower dashed line. Escape was

472 common ( $>60 \%)$ if $t_{e} / t_{s}<<1$, corresponding to the region above the upper dashed

473 line. 

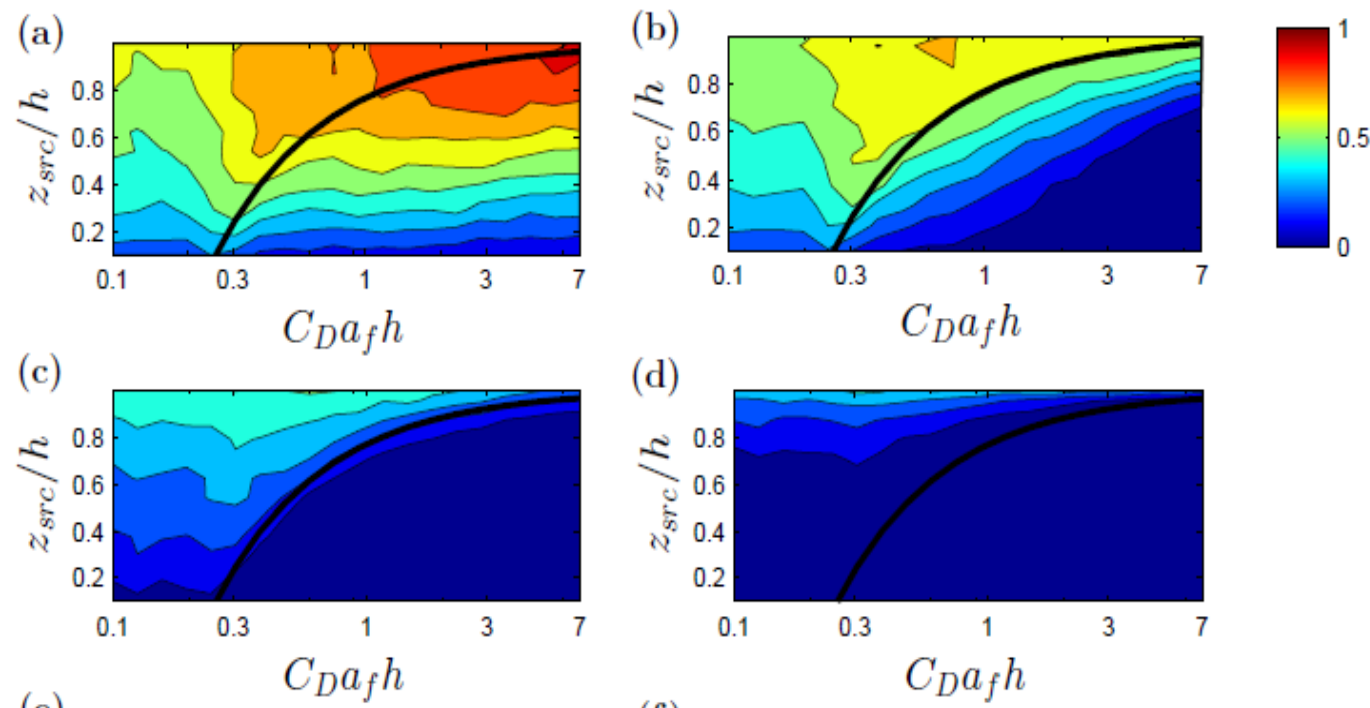

(e)
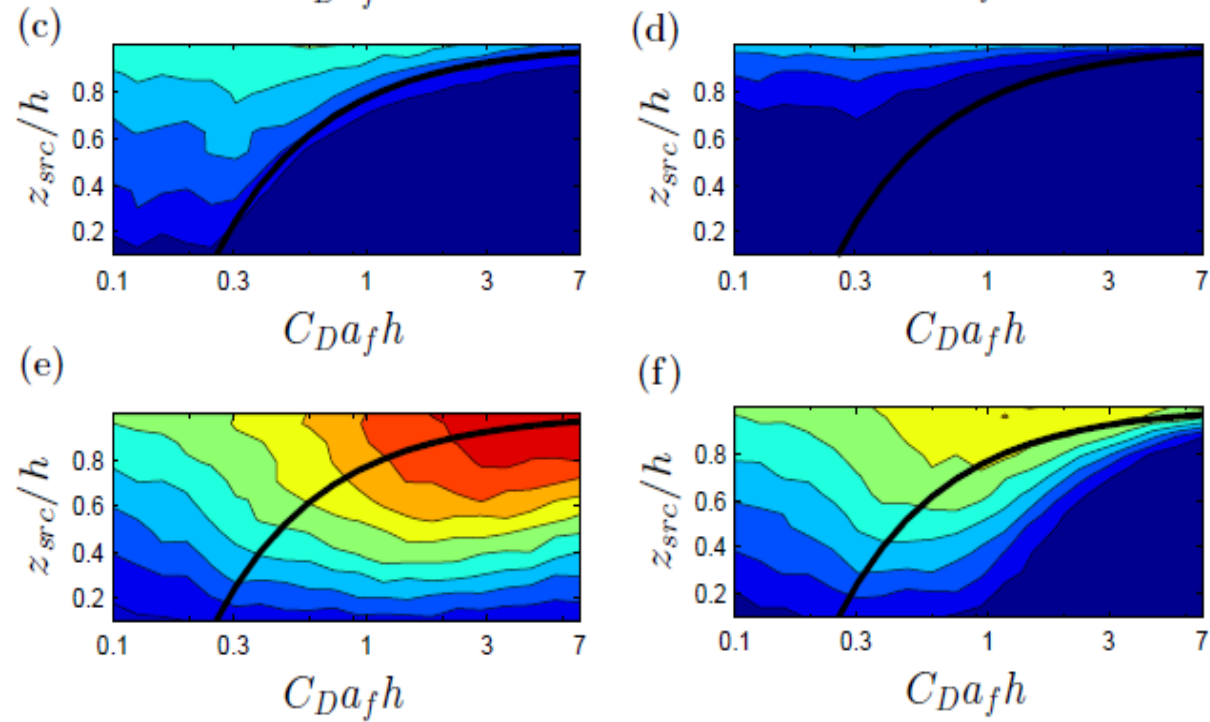

(f)

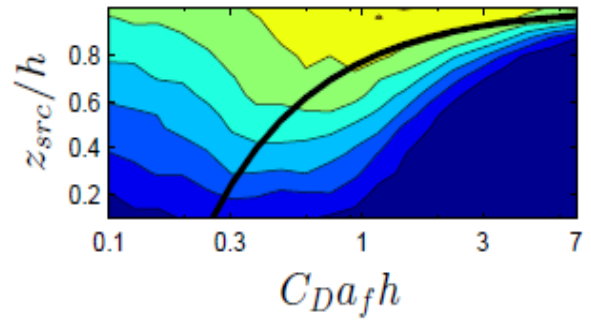

$4:$

475 Figure 5 Escape fraction ( $E F=0$ to 1, as shown in color bar) as a function of source height $z_{s r c}$ 476 normalized by canopy height $h$ and non-dimensional canopy density $C_{D} a_{f} h$. The penetration of 477 turbulence from above extends to $z=h-\delta_{e}$, denoted by the thick black line in each subplot.

478 For (a) to (d), $h=2.1 \mathrm{~m}$ with varying $a_{f}$, and $w_{s} / u_{*}=0(\mathrm{a}), w_{s} / u_{*}=0.1$ (b), $w_{s} / u_{*}=1$ (c), $479 w_{s} / u_{*}=5(\mathrm{~d})$. For (e) and (f), $a_{f}=0.79 \mathrm{~m}^{-1}$ with varying $h$, and $w_{s} / u_{*}=0(\mathrm{e}), w_{s} / u_{*}=0.1$ (f). 

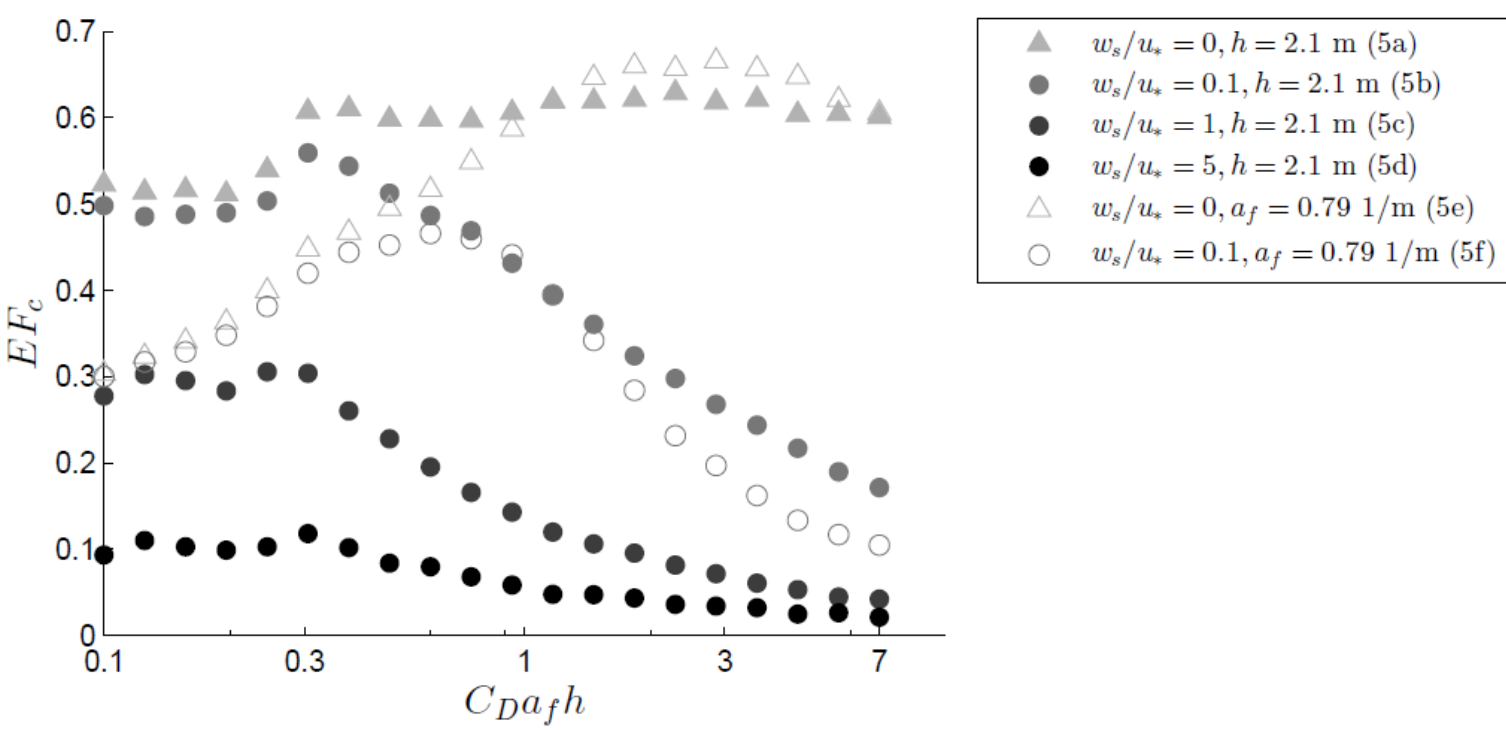

481

Figure 6 Canopy average escape fraction $\left(E F_{c}\right)$ for canopies shown in Figure 5 , as a function of

483 the non-dimensional canopy density $C_{D} a_{f} h=0.1-7$. Filled symbols denote canopies with $h=2.1 \mathrm{~m}$ and varying $a_{f}$; open symbols denote canopies with $a_{f}=0.79 \mathrm{~m}^{-1}$ and varying $h$.

Next, RDM was used to examine escape fraction over a range of canopy densities (Figure 5). Four values of velocity ratio were considered $\left(w_{s} / u_{*}=0,0.1,1,5\right)$. To explore the individual influences of $a_{f}$ and $h$, Figures $5 a$ through $5 d$ hold canopy

488 height constant $(h=2.1 \mathrm{~m})$ and vary $a_{f}=0.07-4.9 \mathrm{~m}^{-1}$, but Figures $5 e$ and $5 f$ hold the 489 frontal area constant $\left(a_{f}=0.79 \mathrm{~m}^{-1}\right)$ and vary $h=0.19-13 \mathrm{~m}$. As expected, the escape 490 fraction decreased with decreasing source height $\left(z_{s r c} / h\right)$ in all cases. In addition, the 491 escape fraction $(E F)$ decreased as the settling velocity ratio $\left(w_{S} / u_{*}\right)$ increased, 492 illustrated by the progression from $w_{s} / u_{*}=0$ to 5 (Figure 5 a to $5 \mathrm{~d}$ ). The trends with canopy density $\left(a_{f} h\right)$ were clearly influenced by the penetration 494 length scale, $\delta_{e}$. The lower limit of the exchange zone $\left(z=h-\delta_{e}\right)$ is indicated in each 495 subplot by a thick black curve. Particles were more likely to escape if they originated 496 above $z=h-\delta_{e}$. This trend was most clear for $w_{s} / u_{*}=0.1$ and 1 (Figures $5 \mathrm{~b}, \mathrm{c}, \mathrm{f}$ ), for 
497 which the contours of escape fraction track the line denoting $\delta_{e}$. A similar relationship

498 would be expected for $z_{m}$, which is also a function of $C_{D} a_{f} h, z_{m}=\frac{1}{2} \delta_{e}$. For $w_{s} / u_{*}=5$

499 (Figure 5d), the pattern was less obvious, because escape fraction was so low over 500 most of the canopy. In this case $\left(w_{s} / u_{*}=5\right)$, escape fraction was $0 \%$ over most of the 501 canopy, but rose to as much as $40 \%$ for particles originating above $h-\delta_{e}$. Generally, 502 for all particles with non-negligible settling velocity $\left(w_{s} / u_{*}=0.1,1,5\right)$, as the depth of 503 the region with elevated turbulence $\left(\delta_{e}\right)$ decreased $\left(C_{D} a_{f} h>0.23\right)$, the canopy average 504 escape fraction also decreased (circles in Figure 6). For these particles, both settling to 505 the ground and capture to the canopy were important mechanisms for detention within 506 the canopy, with the region $z_{s r c} / h \geq 0.5$ dominated by deposition on the canopy, and 507 the region $z_{s r c} / h \leq 0.5$ dominated by settling to the ground (data not shown). Neutrally buoyant particles $\left(w_{s} / u_{*}=0\right)$ exhibited different escape behavior 509 (Figures 5a, f), because these particles could only deposit to the ground or to the 510 canopy via turbulent diffusion. For neutrally buoyant particles, the canopy average 511 escape fraction increased with increasing canopy density (triangles in Figure 6). This 512 can be explained by the trends in deposition by turbulent diffusion. At low values of $513 C_{D} a_{f} h$, diffusivity was elevated across the full canopy height (Figure 2), so that particles 514 from any source height could reach the ground and deposit by diffusion. As $C_{D} a_{f} h$ 515 increased, diffusivity within the canopy decreased (Figure 2), with a coincident decrease 516 in deposition to the ground, leaving more particles available to escape, so that canopy 517 average escape increased. 
The trends in escape fraction were similar for constant $h$ and constant $a_{f}$. For

519 example, the difference in escape fraction $(\Delta E F)$ between each $\left(C_{D} a_{f} h, z_{s r c} / h\right)$ pair in 520 Figures $5 \mathrm{a}$ (constant $h$ ) and $5 \mathrm{e}$ (constant $a_{f}$ ) was $\Delta E F=0.06 \pm 0.006(\mathrm{SE})$; between

521 Figures $5 \mathrm{~b}$ (constant $h$ ) and $5 f$ (constant $a_{f}$ ), the difference in escape fraction was $522 \Delta E F=0.08 \pm 0.006$ (SE). This demonstrated that escape was more strongly dependent 523 on the non-dimensional parameter $\left(C_{D} a_{f} h\right)$ than on the individual parameters of $a_{f}$ and $524 h$. However, secondary influences from the individual contributions of $a_{f}$ and $h$ were 525 apparent at low canopy density. Specifically, for $C_{D} a_{f} h \leq 0.5$ higher escape fractions 526 were observed for the canopies with constant $h=2.1 \mathrm{~m}$ (Figure 6, solid symbols) than 527 for the canopies with constant $a_{f}=0.79 \mathrm{~m}^{-1}$, for which $h=0.19-0.93 \mathrm{~m}$ over the 528 range $C_{D} a_{f} h=0.1-0.5$ (Figures 6 , open symbols). In other words, for the same value 529 of $C_{D} a_{f} h$ higher escape fraction was observed for the taller canopy. This may be

530 explained by the difference in the pseudo-velocity term $\left(\frac{d K_{z}}{d z}\right)$ in eq. (2), which was larger 531 for the taller canopies (data not shown). We caution, however, that this effect may arise 532 from the oversimplified representation of the vertical profile of diffusivity. More detailed 533 measurements of diffusivity profiles are needed to consider the real impact of the 534 pseudo-velocity.

\section{Conclusion}

Forecasting infections from fungal disease can facilitate a reduction of fungicide application while maintaining crop yield (Aylor, 1999). This paper presented a practical 
539 tool for predicting spore escape fraction, a required input to forecast the long-range 540 transport of spores. The proposed RDM predicted escape fraction from a simple set of

541 parameters [canopy height, canopy density, spore settling velocity, vegetation length

542 scale, and wind speed] and did not require detailed velocity or turbulence

543 measurements as input. The model was validated against field measurements of spore

544 concentration downwind from a source within a maize canopy, and it was shown to 545 perform as well as, or better than, more complex LES and LSM models. Although the 546 RDM did not explicitly represent individual turbulent events (sweeps and ejections), it 547 can predict escape fraction, because maximum particle escape, which was used to 548 define escape fraction, occurred in the far-field, that is after transport over several 549 integral time scales. The RDM demonstrated that escape fraction increased as canopy 550 density $\left(a_{f} h\right)$ decreased, as the settling velocity ratio $\left(w_{s} / u_{*}\right)$ decreased, and as the 551 source height $\left(z_{s r c} / h\right)$ increased, confirming earlier studies (Aylor, 1990, Aylor, 1989, 552 Aylor, 1999, Gleicher et al., 2014). The influence of the canopy density was largely 553 reflected in the penetration length scale $\left(\delta_{e}\right)$, which segregated the canopy into regions 554 of high and low escape probability. As a canopy matures, both canopy height and LAI 555 increase, decreasing $\delta_{e} / h$ and creating a larger region within the lower canopy from 556 which spore escape is inhibited. 


\section{Acknowledgements:}

This material is based upon work supported by the National Science Foundation under Grant No. AGS-1005480. Any opinions, findings, or recommendations expressed in this material are those of the authors and do not necessarily reflect the views of the National Science Foundation

\section{Works Cited}

Andrade, D., Zaitao, P., Dannevik, W., and Zidek, J., 2009. Modeling soybean rust spore escape from infected canopies: Model description and preliminary results. J Appl Meteor Climatol 48, 789-803.

Aylor, D.E., 1982. Modeling spore dispersal in a barley crop. Agric Meteorol 26, 215219.

Aylor, D.E., 1986. A framework for examining the inter-regional aerial transport of fungal spores. Agric For Meteorol 38, 263-288. Boundary-Layer Metorol 46, 257-273.

Aylor, D.E., 1989. Dispersion of spores released from an elevated line source within a wheat canopy.

Aylor, D.E., 1990. The role of intermittent wind in the dispersal of fungal pathogens. Annu Rev Phytopathol 28, 73-92.

Aylor, D.E., 2005. Quantifying maize pollen movement in a maize canopy. Agric For Meteorol 131, 247-256.

Aylor, D.E., 1999. Biophysical scaling and the passive dispersal of fungus spores: relationship to integrated pest management strategies. Agric For Meteorol 97, 275-292.

Aylor, D.E., Flesch, T.K., 2001. Estimating spore release rates using a Lagrangian stochastic simulation model. J Appl Meteorol 40, 1196-1208.

Aylor, D.E., Irwin, M.E., 1999. Aerial dispersal of pests and pathogens: implications for integrated pest management. Agric For Meteorol 97, 233-234.

Aylor, D.E., Taylor, G.S., 1983. Escape of Peronospora tabacina spores from a field of diseased tobacco plants. Phytopathol 73, 525-529.

Boughton, B.A., Delaurentis, J.M., 1987. A stochastic model of particle dispersion in the atmosphMere. Boundary-Layer Meteorol 40 (1), 147-163.

Chang, J.C., Hanna, S.R., 2004. Air quality model performance evaluation. Meterol Atmos Phys 87, 167-196.

Chamecki, M., 2013. Persistence of velocity fluctuations in non-Gaussian turbulence within and above plant canopies. Phys Fluids 25, 115110. 
Denmead, O.T., Bradley, E.F., 1987. On scalar transport in plant canopies. Irrigation Sci

$5978,131-149$.

598 Durbin, P.A., 1983. Stochastic differential equations and turbulent dispersion. NASA 599 Reference Publication 1103.

600 Elskus, A.A., 2012. Toxicity, sublethal effects, and potential modes of action of select 601 fungicides on freshwater fish and invertebrates. U.S. Geological Survey Open-File 602 Report 2012-2013, at http://pubs.usgs.gov/of/2012/1213/.

603 Finnigan, J.J., 2000. Turbulence in plant canopies. Annu Rev Fluid Mech 32, 519-571.

604 Ghisalberti, M., 2009. Obstructed shear flows: similarities across systems and scales. J 605 Fluid Mech 641, 51-61.

606 Ghisalberti, M., Nepf, H., 2005. Mass transport in vegetated shear flows. Env Fluid 607 Mech 5, 527-551.

608 Ghisalberti, M., Nepf, H., 2006. The structure of the shear layer in flows over rigid and 609 flexible canopies. Env Fluid Mech 6, 277-301.

Gleicher, S., Chamecki, M., Isard, S.A., Pan, Y., Katul, G.G., 2014. Interpreting threedimensional spore concentration measurements and escape fraction in a crop canopy using a coupled Eulerian-Lagrangian stochastic model. Agricultural and Forest Meteorology 194, 118-131.

Grimmond, C.S.B., and Oke, T.R., 1999. Aerodynamic properties of urban areas derived from analysis of surface form. J Appl Meterorol 38 (9), 1262-1292.

Hanna, S.R., 2003. Uncertainties in air quality model predictions. Boundary Layer Meteorol 62: 3-20.

Harman, I.N., Finnigan, J.J., 2007. A simple unified theory for flow in the canopy and roughness sublayer. Boundary-Layer Meteorol 123 (2), 339-363.

Hassid, S., 1983. Turbulent Schmidt number for diffusion models in the neutral boundary layer. Atmos. Environ. 17(3), 523-527.

Isard, S.A., Russo, J.M., Ariatti, A., 2007. The integrated aerobiology modeling system applied to the spread of soybean rust into the Ohio River valley during September 2006. Aerobiologia 23, 271-282.

Israelsson, P.H., Y.D. Kim, E.E. Adams, 2006. A comparison of three Lagrangian approaches for extending near field mixing calculations. Environ Model Softw 21, 16311649.

de Jong, M.D., 1992. Risk assessment for the application of biological control of a forest weed by a common plant pathogenic fungus. Risk Analysis 12, 465-466. 
647 648

649 650

651 652

653 654

655 656 657

658

659

660

661

662

663

664

665

666 de Jong, M.D., Wagenmakers, P.S., Goudriaan, J., 1991. Modelling the escape of Chondrostereum purpureum spores from a larch forest with biological control of Prunusserotina. Netherlands Journal of Plant Pathology 97, 55-61.

Koeltzsch, K., 2000. The height dependence of the turbulent Schmidt number within the boundary layer. Atmos. Environ. 34, 1147-1151.

Launder, B., 1976. Topics in Applied Physics, Vol. 12, Chapt. 6. Heat and Mass Transport. Springer-Verlag, 231-287.

Legg, B.J., and Powell, F.A., 1979. Spore dispersal in a barley crop: a mathematical model. Agric. Meterorol., 20: 47-67.

Luhar, M., Rominger, J., H. Nepf, 2008. Interaction between flow, transport, and vegetation spatial structure. Environ Fluid Mech 8, 423-439.

Massman, W.J., Weil, J.C., 1999. An analytical one-dimensional second-order closure model of turbulence statistics and the Lagrangian time scale within and above plant canopies of arbitrary structure. Boundary-Layer Meteorol 92, 81-107.

Nathan, R., Katul, G.G., 2005. Foliage shedding in deciduous forests lifts up longdistance seed dispersal by wind. Proc. Natl. Acad. Sci. U.S.A. 102 (23), 8251-8256.

Nepf, H.M., 2012. Flow and transport in regions with aquatic vegetation. Annu Rev Fluid Mech 2012 44, 123-142.

Nepf, H.M., M. Ghisalberti, B. White, E. Murphy, 2007. Retention time and dispersion associated with submerged aquatic canopies. Water Res Res 43, W04422.

Pan, Y., Chamecki, M., Isard, S.A., 2014. Large-eddy simulation of particle dispersion inside the canopy roughness sublayer. J Fluid Mech 753, 499-534.

Poggi, D., A. Porporato, L. Ridolfi, J. D. Albertson, G.G. Katul, 2004. The effect of vegetation density on canopy sub-layer turbulence. Boundary-Layer Meteorol 111, 565587.

Raupach, M., 1994. Simplified expressions for vegetation roughness length and zeroplane displacement as functions of canopy height and area index. Boundary-Layer Meteorol 71, 211-216.

Raupach, M.R., Finnigan, J.J., Brunet, Y., 1996. Coherent eddies and turbulence in vegetation canopies: the mixing-layer analogy. Boundary-Layer Meteorol 78, 351-382.

Roberts, J.R., Reigart, J.R., 2013. Recognition and management of pesticide poisonings. $6^{\text {th }}$ ed. United States Environmental Protection Agency, Office of Pesticide Programs, 272 pp. Available http://www2epa.gov/pesticide-worker-safety/recognitionand-management-pesticide-poisonings 
667 Silva, P.S.L., Silva, K.M.B., Silva, P.I.B., Oliveira, V.R., Ferreira, J.L.B., 2012. Green ear 668 yield and grain yield of maize cultivars in competition with weeds. Planta Daninha 28

669 (1), 77-85.

670 Schlichting H., 1936. Experimental investigation of the problem of surface roughness.

$671 \quad$ NACA Technical Memorandum No 823.

672

673

Shaw, R.H., Tavangar, J., Ward, D.P., 1983. Structure of the Reynolds stress in a

674 canopy layer. J Clim Appl Meteorol 22, 1922-1931.

675 Tanino, Y. and Nepf, H.M., 2008. Lateral dispersion in random cylinder arrays at high 676 Reynolds number. J Fluid Mech 600, 339-371.

677 Thom, A., 1971. Momentum absorption by vegetation. QJR Meteorol Soc 97, 414-428.

678 Thomas, C., Foken, T., 2007. Flux contribution of coherent structures and its 679 implications for the exchange of energy and matter in a tall spruce canopy. Boundary680 Layer Meteorol 123, 317-337.

681 Wilson, J.D., Sawford, B.L., 1996. Review of Lagrangian stochastic models for 682 trajectories in the turbulent atmosphere. Boundary-Layer Meteorol 78, 191-210.

683 Wilson, J.D., Ward, D.P., Thurtell, G.W., Kidd, G.E., 1982. Statistics of atmospheric 684 turbulence within and above a corn canopy. Boundary-Layer Meteorol 24, 495-519.

685 Wilson, J.D., Yee, E., 2007. A critical examination of the random displacement model of 686 turbulent dispersion. Boundary-Layer Meterorol 125, 399-416.

687 Wilson, J.D., 2013. Turbulent Schmidt numbers above a wheat crop. Boundary-Layer 688 Meteorol 148, 255-268. 